\title{
Neuro-Behçet's disease presenting as isolated intracranial hypertension
}

\begin{abstract}
Dear Editor,
Behçet's disease (BD) is a chronic multisystem inflammatory disease with a classic triad of painful oral ulcers, genital ulcers and uveitis. Neurological manifestations, though uncommon, can affect both central and peripheral nervous system; producing parenchymal, non-parenchymal and mixed forms of the disease. ${ }^{1}$ Ophthalmic findings include ocular inflammation and other neuro-ophthalmic signs secondary to neuro-Behçet's (NB).
\end{abstract}

In this report, we discuss a patient who was initially treated for idiopathic intracranial hypertension (IIH), and was later found to have raised intracranial pressure (ICP) secondary to NB, without evidence of cerebral venous sinus thrombosis (CVST).

A 38-year-old Turkish man who resides overseas presented with left eye blurring of vision for 3 months. Prior to this, he had experienced floaters, flashes, and intermittent visual obscurations over the same eye for 1-2 years. He had mild headaches for a week, not requiring analgesia. Magnetic resonance venography (MRV) was performed overseas in view of his symptoms, and was normal. Apart from a previous ear infection, he had no other medical history or medication use. On examination, best-corrected visual acuities (BCVAs) were 20/20 on the right and 20/30 on the left. His intraocular pressures and anterior segment examination were normal. There was a mild relative afferent pupillary defect on the left, and he read 14/15 plates on Ishihara testing in the left eye compared with all 15 plates on the right. Fundus examination revealed diffuse left optic disc swelling and mild superonasal swelling of the right optic disc. There were no vitreous cells, haemorrhages, exudates, vasculitis or macular oedema. Static automated perimetry of the left eye showed temporal visual field defects but was normal for the right. He underwent magnetic resonance imaging (MRI) of the brain with contrast, which was normal apart from a partially empty sella. Lumbar puncture showed raised opening pressures (OP) of 35.4 $\mathrm{cmH}_{2} \mathrm{O}$, and his cerebrospinal fluid (CSF) composition and cultures were normal. He was treated for IIH with oral acetazolamide $250 \mathrm{mg}$ twice daily and subsequently managed overseas, where his medications were gradually tapered and stopped over weeks as his symptoms resolved.

A year later, he returned with bilateral worsening vision despite oral acetazolamide $250 \mathrm{mg}$ twice daily, which had been prescribed by his primary physician for the complaint of headaches that began 6 weeks prior to re-presentation. BCVAs were $20 / 150$ on the right and 20/40 on the left. Fundus examination showed bilateral disc swelling, mild vitritis and areas of subretinal fluid, with enlarged blind spots seen on perimetry. Anterior segment examination was normal. On further questioning, he admitted to recurrent oral ulcers 3-4 times a year for more than 15 years, and 2 episodes of scrotal ulcers over the last 2 years. In addition, he had intermittent red spots on his shin and non-specific knee pain, which raised the suspicion of BD. Repeat MRI and MRV showed distension of both optic nerve sheaths (Fig. 1A) and an empty sella (Fig. 1B) consistent with raised ICP. There was no enhancement of the optic nerves (Fig. 1C), and no CVST was found on angiography (Fig. 1D). Extensive investigations including serology for autoimmune diseases and infections were unremarkable. Significantly, repeat lumbar puncture revealed raised OP of $33 \mathrm{cmH}_{2} \mathrm{O}$ and raised proteins of $0.61 \mathrm{~g} / \mathrm{L}$. CSF cell counts and cultures were normal. He was also positive for HLA-B51.

He was treated with intravenous methylprednisolone $1 \mathrm{~g} /$ day for 3 days, followed by a gradual oral taper, with acetazolamide for the raised ICP. In view of the anticipated need for long-term immunosuppression, he was started on azathioprine. Over 6 months, BCVAs improved to $20 / 30$ on the right and $20 / 20$ on the left, and there was resolution of optic disc swelling and macular oedema.

$\mathrm{NB}$ is an uncommon manifestation of BD affecting $<10 \%$ of patients with the disease. ${ }^{1,2}$ Non-parenchymal disease variant can present with strokes, cognitive dysfunction, psychiatric disorders, and intracranial hypertension secondary to CVST. ${ }^{3}$ Apart from raised OP, CSF is typically normal in CVST. This is in contrast to cases of parenchymal disease, which may display CSF leukocytosis as well as raised proteins and interleukin-6, signifying inflammation. Mixed forms of NB are less common than parenchymal disease or non-parenchymal involvement with CVST.

Headache and visual symptoms secondary to CVST, migraine spectrum disorders, tension headaches, pain from uveitis and complications of cerebral aneurysms may affect up to $70 \%$ of patients with NB. ${ }^{1}$ However, in up to $30 \%$ of patients ${ }^{4}$ who first present with CVST, the diagnosis of BD was unknown, which could lead to a delay in treatment till other clinical features manifest. 


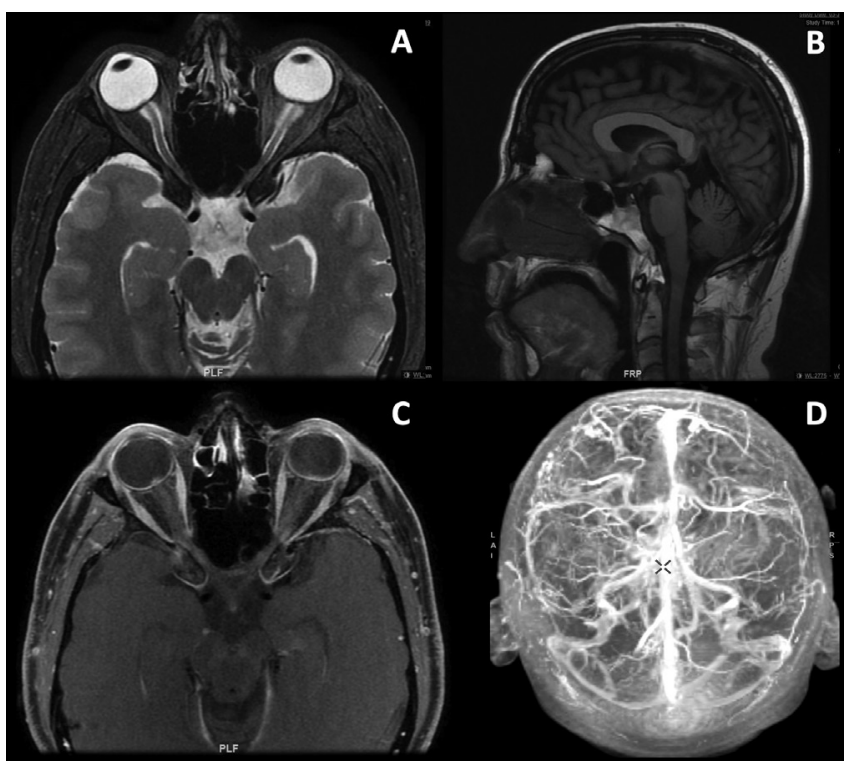

Fig. 1. Neuroimaging showing signs suggestive of raised intracranial pressure.

(A) Distension of optic nerve sheath on T2-weighted magnetic resonance imaging scan. (B) Sagittal section showing the presence of an empty sella. (C) Lack of optic nerve enhancement to suggest neuritis. (D) Angiogram demonstrating good flow through the cerebral veins and venous sinuses.

Papilloedema in patients with BD was first described in $1959^{5}$ and largely attributed to CVST, based on the prevalence of thrombophlebitis associated with the disease. Akman et al. reported a series of 16 patients with known BD who suffered headaches and vomiting secondary to papilloedema, with absence of mass lesions on neuroimaging. ${ }^{6}$ In all but 1 of the patients in the series, CVST was found either at initial work-up, or during further investigations due to recurrence of symptoms. Interestingly, these patients suffered more recurrences and had lower incidence of uveitis.

Our patient had an atypical course, as initially, MRI and CSF composition were normal. Isolated elevated ICP was consistent with the diagnosis of IIH by the Modified Dandy Criteria. However, IIH typically occurs in obese females, ${ }^{7}$ with risk factors including oral contraceptive or steroid use. Development of mild posterior uveitis and worsening disc swelling despite acetazolamide, prompted the clinician to re-investigate. Further clinical history clinched the diagnosis of BD as defined by the International Criteria for Behçet's Disease (ICBD). In addition, he was positive for HLA-B51, which is historically known to have a primary and causal risk determinant for $\mathrm{BD} .{ }^{8}$ Although raised ICP in known BD has been described, this can be confounded by steroid treatment, which predisposes to IIH. Our patient was not known to have BD nor steroid intake, and subsequently had an unusual combination of active uveitis and raised ICP without evidence of CVST after repeated investigations.

In conclusion, the varying presentations of NB can be misleading, especially in patients without a background history of BD. CVST is the commonest cause of intracranial hypertension in NB and appropriate imaging studies should be performed, and repeated, if there is clinical evidence of raised ICP. Although uncommon, our patient has demonstrated that it is possible to have concurrent uveitis and disc swelling from raised ICP without CVST, providing us with challenges in diagnosis and management.

\section{REFERENCES}

1. Al-Araji A, Kidd DP. Neuro-Behçet's disease: epidemiology, clinical characteristics, and management. Lancet Neurol 2009;8:192-204.

2. Cheng YK, Thong BY, Chng HH. Behçet's Disease: Experience in a Tertiary Rheumatology Centre in Singapore and a Review of the Literature. Ann Acad Med Singap 2004;33:510-4.

3. Kidd DP. Neurological complications of Behçet's syndrome. J Neurol 2017;264:2178-83.

4. Aguiar de Sousa D, Mestre T, Ferro JM. Cerebral venous thrombosis in Behçet's disease: a systematic review. J Neurol 2011;258:719-27.

5. Masheter HC. Behçet's syndrome complicated by intracranial thrombophlebitis. Proc R Soc Med 1959;52:1039-40.

6. Akman-Demir G, Bahar S, Baykan-Kurt B, et al. Intracranial hypertension in Behcet's Disease. Eur J Neurol 1996;3:66-70.

7. Daniels AB, Liu GT, Volpe NJ, et al. Profiles of obesity, weight gain, and quality of life in idiopathic intracranial hypertension (pseudotumor cerebri). Am J Ophthalmol 2007;143:635-41.

8. de Menthon M, Lavelley MP, Maldini C, et al. HLA-B51/B5 and the Risk of Behçet's Disease: A Systematic Review and MetaAnalysis of Case-Control Genetic Association Studies. Arthritis Rheum 2009;61:1287-96.

Hazel A $\underline{\text { Lin, }}{ }^{1}$ MBBS, MMed (Ophth),

David Z Chen, ${ }^{1}$ MBBS, MMed (Ophth), FRCOphth,

Clement WT Tan, ${ }^{1}$ MBBS, MMed (Ophth), FRCSEd

${ }^{1}$ Department of Ophthalmology, National University Hospital, Singapore

Correspondence: Dr Hazel Anne Lin, Department of Ophthalmology, National University Hospital, 1E Kent Ridge Rd, Singapore 119228.

Email: hazel_anne_lin@nuhs.edu.sg 